\title{
Why and how to predict sea level changes at a tide gauge station with prediction intervals
}

https://doi.org/10.1515/jogs-2018-0012

Received March 3, 2018; accepted November 16, 2018

\begin{abstract}
Predicting sea level rise is essential for current climate discussions. Empirical models put in use to monitor and analyze sea level variations observed at globally distributed tide gauge stations during the last decade can provide reliable predictions with high resolution. Meanwhile, prediction intervals, an alternative to confidence intervals, are to be recognized and deployed in sea level studies. Predictions together with their prediction intervals, as demonstrated in this study, can quantify the uncertainty of a single future observation from a population, instead of the uncertainty of a conceivable average sea level namely a confidence interval, and it is thereby, better suited for coastal risk assessment to guide policy development for mitigation and adaptation responses.
\end{abstract}

Keywords: Prediction; Prediction intervals; Best linear unbiased prediction; Confidence intervals; Projection; Tide gauges; Climate change; Risk assessment

Don't cross a river if it is four feet deep on average. Nassim Nicholas Taleb (2007).

\section{Introduction}

A projection is associated with the possibility of something happening given a certain set of plausible, but not necessarily probable circumstances (Bray and Storch 2009). A projection is a broad prediction where the extrapolated values are subject to certain assumptions (Gabriele and Freichter, 2011). In the context of sea level studies, projections are explanatory through their predicates, which include thermal expansion expected to contribute to more than half of the long-term sea level rise, eustatic effects

*Corresponding Author: H. Bâki Iz: Division of Geodetic Science, School of Earth Sciences, The Ohio State University, Columbus, Ohio, USA, E-mail: H.Baki.Iz@gmail.com caused by the increasingly rapid loss of land ice overtime, as the underlying causal relationships.

Projections are safe because they are imprecise: all that is projected is the direction of the underlying effects. Also, projections are not easily testable by observations because of their large uncertainties they carry forward (ibid). Their predictive powers are heavily dependent on the performance of their predicates. The InterGovernmental Panel on Climate Change (IPCC), for instance, projects global sea level to reach between half a meter and a meter of sea-level rise by the end of the 21st century with a rate greater than the global sea level rise experienced during the 20th century (IPCC, 2013) for an unmitigated future rise in emissions (RCP8.5) accompanied by a broad brush of probabilities (Mastendra et al., 2010).

In contrast, sea level predictions ${ }^{1}$ rely heavily on past data such as sea level measurements at tide gauge stations as they are reconstructed in this study with the aid of a harmonic model (a trigonometric model) consisting predominantly of a secular sea level trend, an acceleration, and periodicities. Sea level predictions assume that sea level variations observed at these stations in recent history, are indicative of their near future kinematics. During the last decade, a series of historically observed cyclic regularities were shown to be caused by the external forcings of the sea level of astronomical origin, such as lunar gravity and their compounding with random or periodic or episodic components of natural sea level variations, such as wind, atmospheric pressure, sea surface temperature, etc. during the $20^{\text {th }}$ century $(\mathrm{Iz}, 2014,2015,2016)$. Because of their exhibited regularities, observed sea level variations are predictable. Meanwhile, sea level trends that were observed at tide gauges are the lumpsum proxies experienced by the oceans induced by regional and global temperature changes (thermosteric effects), land ice, and their interactions, and further related to climate change variables such as global natural and anthropogenic $\mathrm{CO}_{2}$ emissions. Their decomposition is inherently difficult to ensure reliable predictions in the long run without additional information. Despite that, quantification and extension of sea

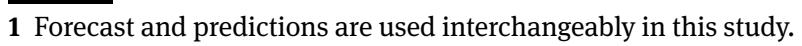


level trends enable assessing the distance between a prediction and projection and future observation of sea levels, and thereby, serve as a reality check for the relevance of projections and their underlying theories. A very low predictability level for predictive models of sea level variations can lead to the development of new measures, alternative theories, new collected data, and new empirical approaches. An explanatory model that is close to a benchmark predictive model may suggest that our understanding of that phenomenon can only be increased marginally. If such a model is very far from the predictive benchmark, this outcome would imply that there are substantial practical and theoretical gains to achieve from further scientific development.

While uncertainties attributed to projections reflect a range of scenarios based on potential outcomes with broadly assigned probabilities, as introduced by the IPCC, which are expected to improve as more information becomes available, statistically well-defined confidence intervals go hand in hand with predictions. A confidence interval of a prediction is a range that is likely to contain the mean response given specified settings of the predictors in the model. In the context of sea level variations, just like the regular confidence intervals, confidence interval of a prediction presents a range of values for the predicted average sea level. This interval, however, does not encapsulate the range or the distribution of individually observable sea levels but simply quantifies the range of their average, i.e. population mean. This distinction is important. For applications such as storm surge heights, predicting sea level at a certain time horizon, assessing extreme events, one needs a prediction interval for the likely realization of a future observation, not a range of a conceivable average sea level namely a confidence interval. This range of random realization of a sea level observable in the future is simply about one of the sampled members of the predicted average sea level. Formally stated, a prediction interval is a range that is likely to contain the response value of a single new observation in the future given specified settings of the predictors in the model with a given probability. Of course, a prediction does not have to be in the future but can also apply to interpolated values within an already observed time series.

Despite the well-established foundations of predictions and prediction intervals in statistical literature, the use of prediction intervals is not prevalent in sea level studies. To fill this gap, this study aims to formulate and demonstrate quantification of a range of random realizations, i.e. the prediction interval, of a future sea level that may be subsequently observed, verified or rejected.
In the following sections, first, the mechanics of the classical linear prediction with confidence intervals will be summarized and extended to include prediction intervals. Subsequently, the corresponding formularies will be demonstrated numerically using Key West USA tide gauge data; first, by establishing a predictive model, then by carrying out a series of predictions together with their confidence and prediction intervals.

\section{Prediction as an extension of linear models, confidence, and prediction intervals}

This section is a brief synthesis of the discussions in Bibby and Toutenburg (1977), Toutenburg (1982), and Goldberger, (1962) regarding prediction and prediction intervals.

Consider the following linear model,

$$
\boldsymbol{y}=\boldsymbol{A x}+\varepsilon, \varepsilon \sim\left(0, \Sigma_{y}\right)
$$

In this expression, there are $n$ observations and $u$ unknown model parameters respectively. The $n \times 1$ vector of observations is denoted by $\boldsymbol{y}$, and $\boldsymbol{A}$ is the $n \times u$, $n>u$ known design (coefficient) matrix of full rank, i.e. $\operatorname{rank}(\boldsymbol{A})=u$. The $u \times 1$ vector of unknown parameters $\boldsymbol{x}$ is assumed to be deterministic in nature. In the above statistical model, the $n \times 1$ vector of disturbances is denoted by $\boldsymbol{\varepsilon}$ with the assumed $E(\boldsymbol{\varepsilon})=\mathbf{0}$, which is the first term within parentheses, whereas the second term $\boldsymbol{\Sigma}_{y}=\sigma^{2} \boldsymbol{W}$ is the full $n \times n$ dispersion matrix of the disturbances (also known as the variance/covariance matrix). The variance of the disturbances in relation to a priori variance of unit weight is represented by $\sigma^{2}$, and $\boldsymbol{W}$ is a known $n \times n$ symmetric matrix.

The estimated unknown parameters $\hat{\boldsymbol{x}}$ are obtained using the least squares solution to Eq. 1 as,

$$
\hat{x}=\left(A^{T} W^{-1} A\right)^{-1} A^{T} W^{-1} y
$$

which is also known as the Best Uniformly Unbiased Estimator (BLUUE), (Iz, 1992). The corresponding $u \times u$ variance-covariance matrix of the estimated parameters, $\boldsymbol{\Sigma}_{\hat{x}}$, is given by,

$$
\boldsymbol{\Sigma}_{\hat{\chi}}=\sigma^{2}\left(\boldsymbol{A}^{\boldsymbol{T}} \boldsymbol{W}^{-1} \boldsymbol{A}\right)^{-\mathbf{1}}
$$

Now, denoting the subscript star to reference predicted values, $n *$ is the number of observations to be predicted. If these observations are generated by the same lin- 
ear model given by Eq. (1), they can be expressed as follows,

$$
\boldsymbol{y}_{\star}=\boldsymbol{A}_{\star} \boldsymbol{X}+\boldsymbol{\varepsilon}_{\star}, \boldsymbol{\varepsilon}_{\star} \sim\left(\mathbf{0}, \boldsymbol{\Sigma}_{\boldsymbol{y}_{\star}}\right)
$$

In this expression, $y_{\star}$ is the $n_{\star} \times 1$ vector of yet to be interpolated, extrapolated, predicted observations and $\boldsymbol{A}_{\star}$ is the $n_{*} \times u$ known design matrix. The statistical properties of the disturbances are carried out over to the predictions with $\boldsymbol{\varepsilon}_{\star}$, which is the $n_{\star} \times 1$ vector of disturbances. Furthermore, it is assumed that the disturbances are uncorrelated, i.e. $E\left(\boldsymbol{\varepsilon} \boldsymbol{\varepsilon}_{*}^{T}\right)=\mathbf{0}$ (alternative derivations are given in Toutenburg, (1982) for the case of correlated disturbances). As before, the $n_{\star} \times n_{\star}$ dispersion matrix of the predicted disturbances is $\boldsymbol{\Sigma}_{y_{\star}}=\sigma^{2} \boldsymbol{W}_{\star}$.

One of the well-known predictors of $\boldsymbol{y}_{\star}$ is given by its least squares estimator (Toutenburg, 1982, pg. 135),

$$
\hat{\boldsymbol{y}}_{\star}=\boldsymbol{A} \star \hat{\boldsymbol{X}}
$$

In this expression, $\hat{\boldsymbol{y}}_{\star}$ is the $n_{\star} \times 1$ vector of predicted observations. Substituting Eq. (2) in Eq. (5) and taking into account Eq. (1),

$$
\begin{aligned}
\hat{y}_{\star} & =A \star \hat{X}=A \star\left(A^{T} W^{-1} A\right)^{-1} A^{T} W^{-1} y \\
& =A \star X+\left(A^{T} W^{-1} A\right)^{-1} A^{T} W^{-1} \varepsilon
\end{aligned}
$$

is obtained. Because $\hat{\boldsymbol{x}}$ is an unbiased estimate of $\boldsymbol{x}$, i.e. $E(\hat{\boldsymbol{x}})=\boldsymbol{x}$, and considering $E(\boldsymbol{\varepsilon})=\mathbf{0}$, the expected value of the predicted $\hat{\boldsymbol{y}}_{\star}$ in the above equation reveals that,

$$
\begin{aligned}
\boldsymbol{E}\left(\hat{\boldsymbol{y}}_{\star}\right) & =\boldsymbol{A}_{\star} \boldsymbol{X}=\boldsymbol{E}\left(\boldsymbol{y}_{\star}\right) \Rightarrow \boldsymbol{E}\left(\hat{\boldsymbol{y}}_{\star}\right)=\boldsymbol{E}\left(\boldsymbol{y}_{\star}\right) \Rightarrow \text { bias : }: \boldsymbol{E}\left(\boldsymbol{y}_{\star}-\hat{\boldsymbol{y}}_{\star}\right) \\
& =\mathbf{0}
\end{aligned}
$$

Therefore, the predictor $\hat{\boldsymbol{y}}_{\star}$ is an unbiased predictor of $y_{\star}$. Using variance propagation, it can be shown that the variance/covariance matrix of the predicted $\hat{\boldsymbol{y}}_{\star}$ denoted by $\boldsymbol{\Sigma}_{\hat{y}_{\star}}$ is given by,

$$
\begin{aligned}
\boldsymbol{\Sigma}_{\hat{\boldsymbol{y}}_{\star}}: & =E\left\{\left(\boldsymbol{y}_{\star}-\mathrm{E}\left(\boldsymbol{y}_{\star}\right)\right)\left(\boldsymbol{y}_{\star}-\mathrm{E}\left(\boldsymbol{y}_{\star}\right)\right)^{\mathrm{T}}\right\} \\
& =\sigma^{2} \boldsymbol{A} \star\left(\boldsymbol{A}^{\boldsymbol{T}} \boldsymbol{W}^{-1} \boldsymbol{A}\right)^{-\mathbf{1}} \boldsymbol{A}_{\star}^{\boldsymbol{T}}
\end{aligned}
$$

The above variance-covariance matrix measures only the spread of $\hat{\boldsymbol{y}}_{\star}$, i.e. its mean square error, from its expected value $\boldsymbol{A} \star \boldsymbol{x}$. In practice however, one needs information about how much predicted values would differ from their actual values, i.e. $\hat{y}_{\star}-y_{*}$. To this end, the corresponding statistic is given by its mean square error of prediction, MSEP, which is defined as follows,

$$
\operatorname{MSEP}\left(\hat{\boldsymbol{y}}_{\star}\right)=\boldsymbol{E}\left\{\left(\hat{\boldsymbol{y}}_{\star}-\boldsymbol{y}_{\star}\right)\left(\hat{\boldsymbol{y}}_{\star}-\boldsymbol{y}_{\star}\right)^{\boldsymbol{T}}\right\}
$$

to be contrasted with its $\boldsymbol{M S E}$,

$$
\operatorname{MSE}\left(\hat{y}_{\star}\right)=\Sigma_{\hat{y}_{\star}}=E\left\{\left(y_{\star}-E\left(y_{\star}\right)\right)\left(y_{\star}-E\left(y_{\star}\right)\right)^{T}\right\}
$$

The narrative of the introduction section regarding the difference between confidence and prediction intervals can now be formally demonstrated by comparing $\operatorname{MSEP}\left(\hat{\mathbf{y}}_{\star}\right)$ with $\boldsymbol{M S E}\left(\hat{\boldsymbol{y}}_{\star}\right)$, where the latter involves the expected value of the predicted value, i.e. its theoretical mean. Considering,

$$
\operatorname{MSE}\left(\hat{\boldsymbol{y}}_{\star}\right)=\boldsymbol{\Sigma}_{\hat{\boldsymbol{y}}_{\star}}=\sigma^{2} \boldsymbol{A} \star\left(\boldsymbol{A}^{\boldsymbol{T}} \boldsymbol{W}^{-1} \boldsymbol{A}\right)^{-1} \boldsymbol{A}_{\star}^{\boldsymbol{T}}
$$

it can be shown that (Toutenburg, 1982, pg. 138, eqs. 5.1.17 - 5.1.19) the MSEP of can be expressed as

$$
\operatorname{MSEP}\left(\hat{y}_{\star}\right)=\Sigma_{\hat{y}_{\star}}+\Sigma_{y_{\star}}-C-C^{T}
$$

where,

$$
\boldsymbol{C}:=\boldsymbol{E}\left\{\left(\hat{\boldsymbol{y}}_{\star}-\boldsymbol{A}_{\star} \boldsymbol{X}\right)\left(\boldsymbol{y}_{\star}-\boldsymbol{A}_{\star} \boldsymbol{X}\right)^{\boldsymbol{T}}\right\}
$$

Because of the assumed $\boldsymbol{E}\left(\boldsymbol{\varepsilon} \boldsymbol{\varepsilon}_{*}^{\boldsymbol{T}}\right)=\mathbf{0}$, that is, future disturbances are not correlated with the disturbances of the observed quantities, it can be shown that $\boldsymbol{C}=\mathbf{0}$, and Eq. (12) reduces to,

$$
\operatorname{MSEP}\left(\hat{y}_{\star}\right)=\boldsymbol{\Sigma}_{\hat{y}_{\star}}+\boldsymbol{\Sigma}_{y_{\star}}=\sigma^{2} \boldsymbol{A}_{\star}\left(\boldsymbol{A}^{T} \boldsymbol{W}^{-1} \boldsymbol{A}\right)^{-1} \boldsymbol{A}_{\star}^{T}+\sigma^{2} \boldsymbol{W}_{\star}
$$

The least squares predictor given by eq. (2) and its prediction error given by Eq. (14) can also be obtained as a special case of a derivation by minimizing the target function given by Eq. (9), (Goldberger, 1962).

Eq. (14) implicates that the difference is always positive definite,

$$
\operatorname{MSEP}\left(\hat{y}_{\star}\right)-\operatorname{MSE}\left(\hat{y}_{\star}\right)>0,
$$

Thereby, prediction intervals are always larger than the confidence intervals.

If the disturbances follow a normal distribution, i.e. $\boldsymbol{\varepsilon} \sim N\left(0, \boldsymbol{\Sigma}_{y}\right)$, then the corresponding confidence interval, $C I$, and the prediction interval, $P I$, for each predicted value are computed from

$$
\begin{array}{r}
C I=\hat{y}_{\star} \pm t_{1-\alpha, D F} r m s e\left(\hat{y}_{\star}\right) \\
P I=\hat{y}_{\star} \pm t_{1-\alpha, D F} r m s e p\left(\hat{y}_{\star}\right)
\end{array}
$$

In these expressions, $t_{1-\alpha, D F}$ is the $t$-score, $D F$ is the degrees of freedom, and $\alpha$ is the significance level. The standard error of the predicted observation is denoted by its root mean square error, rmse, and root mean square error of the prediction, rmsep. Student's $t$-distribution, which approximates the counterpart of the normal distribution of the underlying population for small samples, 
can be replaced by the normal distribution when the number of observations is large.

Note that the variance of the disturbances, $\sigma^{2}$ in the above expression, is an a priori value given prior to the least squares solution to Eq. 1 . An $F$-test for the equality of the variances for the a posteriori value $\hat{\sigma}^{2}$ (the estimated variance for the disturbances) needs to be carried out to decide whether the $a$ priori or $a$ posteriori variance is to be used in calculating prediction and confidence intervals. Because the predictions are yet to be realized observations, a priori or a posteriori variance need to be used consistently for the observed as well as predicted disturbances. In practice, normal distribution assumption about the disturbances, in particular, the assumption that the expected value of the disturbances being zero, may fail because of the unmodeled systematic effects. Therefore, proper scrutiny of the residuals is always needed.

The ongoing brief discussion and the formularies so far can be quantified through the following example. In the following section, first, a predictive model is established, which will be subsequently used to carry out a series of predictions with confidence and prediction intervals.

\section{A predictive model for Key West sea level variations}

Iz (2014, 2015) developed a harmonic model consisting of a secular trend, acceleration, and statistically significant, globally prevalent, specific periodicities in sea level variations that are due to the coupling of external forcing of luni-solar origin, in tandem with other natural or forced sea level variations and broadband internal ocean-atmosphere interactions, including atmospheric and thermosteric contributions producing signatures at multi-decadal time scales (sub and super harmonics). The model reads as,

$$
\begin{aligned}
h_{t}= & h_{t_{0}}+v\left(t-t_{0}\right)+\frac{a}{2}\left(t-t_{0}\right)^{2}+\sum_{k=0}^{n}\left\{\alpha_{k} \sin \left[\left(\frac{2 \pi}{P_{k}}\right)\left(t-t_{0}\right)\right]\right. \\
& \left.+\gamma_{k} \cos \left[\left(\frac{2 \pi}{P_{k}}\right)\left(t-t_{0}\right)\right]\right\}+\varepsilon_{t}
\end{aligned}
$$

In this model, the initial epoch of the measurements is shifted to the middle of the series for shorter time offsets to improve the numerical stability of the solutions where, $h_{t}$ represents the monthly averaged tide gauge data at $t=$ $t_{\text {Start }} \ldots t_{\text {End }}$, and $h_{t_{0}}$ is the unknown sea level reference height defined at the middle epoch of the measurements. The initial velocity is $v$, and $a$ is the acceleration or deceleration in sea level.
In addition, sea level changes at a given station exhibit multi-decadal scale sea level variations caused by the interaction of the tidal effects due to the regression of the lunar node, which completes its cycle in $P=18.613 \mathrm{yr}$. The compounding of the nodal tide with natural persistent patterns of sea level changes induced by atmospheric pressure variations, wind patterns, and broadband internal ocean-atmosphere interactions produces sea level variations at multi-decadal time scales with periods $2 \times P=37.226$ yr., $3 \times P=55.839$ yr., $5 \times P=74.452$ yr., $\ldots$ (subharmonics), and its super harmonics with periods $P / 2=9.306 \mathrm{yr} ., P / 3=$ 6.204 yr.... (Iz, 2014, Keeling and Worf, 1997, and Munk et al., 2002). Potential variations in total solar radiation with a period of $P=11.1$ yr. may also generate subharmonics such as with a period, $2 \times P=22.2 \mathrm{yr}$. In Eq. 18, the summation is carried over for all the above harmonics $n$, listed in Table 1 . These sub and super harmonics are statistically significant in global sea level variations as shown by Iz (2014).

A recent study by Iz (2015) detected and quantified that some of the globally distributed tide gauge stations were also affected by a multitude of periodic changes at decadal scales including a statistically significant $(p<$ $0.05)$ periodicity within the $12-14$ yr. range. This effect is also incorporated into the model given by Eq. 18 .

Table 1: Periods (yr.) of the Luni-Solar compounders and decadal cyclicity modeled in the harmonic model.

\begin{tabular}{lllllll}
\hline Nodal & Nodal & Nodal & Solar & Annual & Chandler & Decadal \\
\hline 74.5 & 18.6 & 3.7 & 11.1 & 1.00 & 1.2 & 12.4 \\
55.8 & 9.3 & 3.1 & 22.2 & 0.50 & & \\
37.2 & 6.2 & 2.6 & & 0.25 & & \\
& 4.7 & 2.3 & & & & \\
\hline
\end{tabular}

The unknown parameters $\alpha_{k}$ and $\gamma_{k}$ are to be estimated and used to calculate the amplitudes and the phase angles of the periodicities. At this point, it is important to emphasize that the model given by eq. (18) is predictive because of its kinematic nature with specific velocity, acceleration and periodicities conceived using past sea level data.

The random variable $\varepsilon_{t}$ represents the lump-sum effect of the random instrument errors and unmodeled effects in sea level changes, i.e., disturbances, which are assumed to be homogeneous and identically distributed with zero expected values. Note that, in general, disturbances of monthly tide gauge data for globally distributed tide gauge stations were demonstrated to be positively autocorrelated (Iz et al., 2012). First order autoregressive random errors (disturbances) alter solution statistics 
markedly if they are not properly accounted for in linear models. However, the first order autocorrelation of the disturbances is negligibly small $(\rho=0.1)$ for the yearly Key West tide gauge data used in this study because of the averaging of monthly data. Note also that annual and sub annual periodicities cannot be estimated using yearly averaged sea level data but are included in the list to be used in subsequent solutions involving monthly averaged tide gauge data.

\section{Key West tide gauge station data}

Key West, USA monthly and yearly tide gauge measurements will be used to predict sea level rise at this station during the $21^{\text {st }}$ century and their corresponding confidence and prediction intervals. Graphical exhibits of the monthly and yearly tide gauge spanning 96 years (1913-2009) are shown in Figure 1 and Figure 2 respectively. All Revised Local Reference (RLR) tide gauge data are referenced to a common datum. The series were downloaded from the Permanent Service for Mean Sea Level (PSMSL) repository, which maintains a tide gauge database from over 1800 stations since 1933 (PSMSL, 2016). No corrections were applied for the Glacial Isostatic Adjustment (GIA); hence, all the inferences will refer to relative sea level changes.

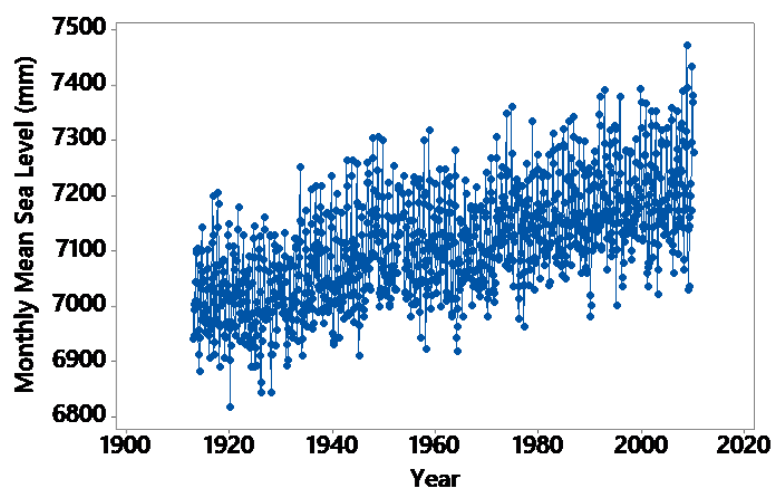

Figure 1: Monthly averaged sea level measurements at Key West, USA tide gauge station (PSMSL, 2016).

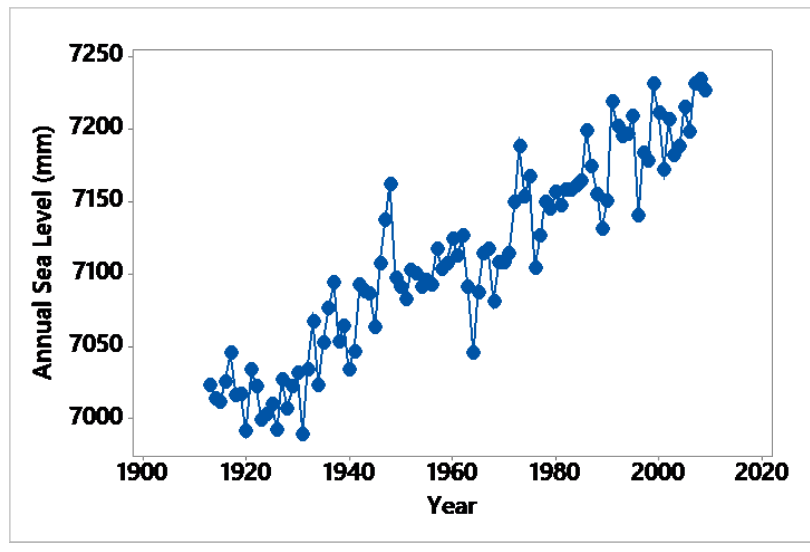

Figure 2: Yearly averaged sea level measurements at Key West, USA tide gauge station (PSMSL, 2016).

\section{Adjusted and predicted sea level rise using short span yearly tide gauge records}

A preliminary solution to the model (Eq. 18) was carried out maintaining only the intercept and the trend parameters using only the recent 25 years of the yearly Key West tide gauge data (the rectangular area shown in Figure 3). This solution will be used as a baseline to assess subsequent more elaborate model solutions with longer data span and high resolution. The short tide gauge data span enables comparisons with satellite altimetry solutions; hence the limitations are informative.

The trend estimate from this model represents the lump-sum effect of steric, eustatic, halosteric, and various local and/or isostatic origin vertical movements experienced by the tide gauge measurements. The rate of sea level rise during this period is estimated to be $2.20 \pm 0.68 \mathrm{~mm} / \mathrm{yr}$. The uncertainty of the trend estimate is large, and the model explains only $28.9 \%$ of the variability in the yearly sea level data during this period at this tide gauge station with an estimated standard error of $24.8 \mathrm{~mm}$ for the residuals.

The corresponding confidence and prediction intervals for a significance level of $\alpha=0.05$ during this period were extended until year 2100 using the relationships given in the previous section. It was assumed that $\boldsymbol{\varepsilon} \sim\left(0, \sigma^{2} \boldsymbol{I}\right)$, and $E\left(\boldsymbol{\varepsilon} \boldsymbol{\varepsilon}_{\star}^{\boldsymbol{T}}\right)=\mathbf{0}$ where $\boldsymbol{I}$ is an identity matrix whose dimensions are defined by the length of the observed ( 25 yrs.) and predicted (90 yrs.) and $\sigma^{2}=1$. As shown in Figure 3, both interval limits diverge rapidly mainly because of the large uncertainty in the estimated trend inferred from a short tide gauge data series ( 25 tide 
gauge yearly averages with 23 degrees of freedom). These statistics will improve markedly in the subsequent solutions with longer series together with the use of modelled periodicities in sea level variations as shown in the following section. Meanwhile, a byproduct of this exercise is a demonstration of potentially large uncertainties caused by short time series, such as those from satellite altimetry, in long term predictions due to the unmodeled periodicities in sea level variations in recent practice (e.g. Nerem et al., 2018).

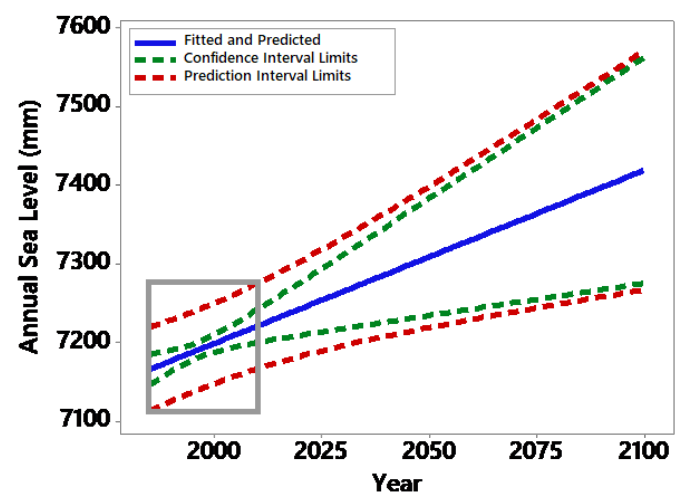

Figure 3: Model solution using only 25 year (1985-2009) yearly Key West tide gauge adjusted observations and their confidence and prediction intervals are are shown within the rectangle $(\alpha=0.05)$. Reference epoch for the solution is 1997. Yearly sea level predictions were extended until year 2100 .
Table 2: Solution statistics: the estimated variance of the disturbances $\hat{\sigma}^{2}=20.53^{2} \mathrm{~mm}^{2}$, Adj $\mathrm{R}^{2}=90.82 \%$. Trend and estimated coefficients that are listed below are in $\mathrm{mm} / \mathrm{yr}$ and $\mathrm{mm}$ respectively. SE refers to the standard error/deviation of the estimated parameters. Only the model parameters that are statsitically significant at $\alpha=0.05$ are listed in the table.

\begin{tabular}{lll}
\hline Parameter & Estimate & SE \\
\hline Constant & 7110.86 & 2.12 \\
Trend & 2.20 & 0.81 \\
Sin $56 \mathrm{yr}$ & -6.84 & 3.04 \\
Sin $37 \mathrm{yr}$ & -8.69 & 2.95 \\
Cos $37 \mathrm{yr}$ & -9.51 & 3.05 \\
Sin $12.4 \mathrm{yr}$ & -6.28 & 2.93 \\
Cos $12.4 \mathrm{yr}$ & 7.29 & 2.98 \\
Cos $6 \mathrm{yr}$ & 10.26 & 2.97 \\
\hline
\end{tabular}

section, the Adj $\mathrm{R}^{2}$ value ${ }^{2}$ of the residuals shows that the harmonic model explains $90.82 \%$ of the yearly sea level variation at this tide gauge station compared to the Adj $\mathrm{R}^{2}$ value of $28.86 \%$ of the trend only model. Improved Adj $\mathrm{R}^{2}$ values are necessary for improved predictive power of a model. In addition, the new model solution is not an overparameterized fit because not only the Adj $\mathrm{R}^{2}$ penalizes the solution for adding new parameters, but it also provides a solution with a larger degree of freedom (88).

Figure 4 shows that the residuals exhibit a normal-like distribution and are free from unmodeled systematic effects. Additional solution statistics, such as the $F$ test and the predicted $R^{2}$ values are indicative of a model with high predictive power.
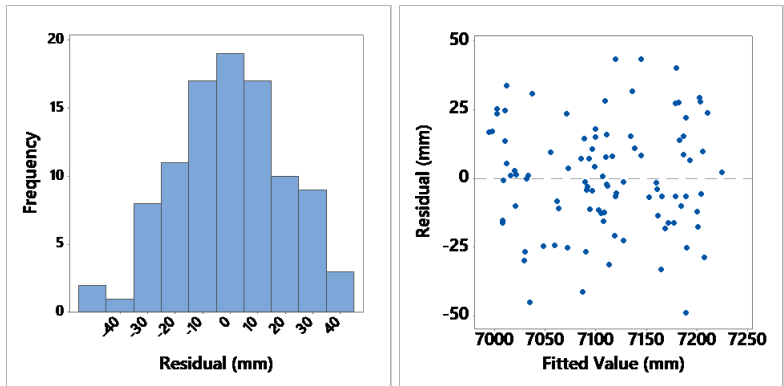

Figure 4: Residual histogram and the residuals vs. adjusted (fitted) yearly sea levels. significant parameters for $\alpha<0.05$. Although the yearly averaged data effectively removed the high frequency variations and reduced the amplitude of the low frequency periodicities, full series revealed that there are statistically significant periodicities listed in Table 2 with their error estimates that cannot be detected using shorter series. Also, even though the trend estimate is practically the same with the trend estimated using shorter series in the previous
2 The adjusted R-squared abbreviated by, is the percent of variation in the observations explained by the model. 
discussed in the previous section. Again, it was assumed that $\boldsymbol{\varepsilon} \sim\left(0, \sigma^{2} \boldsymbol{I}\right), \boldsymbol{\varepsilon}_{\star} \sim\left(0, \sigma^{2} \boldsymbol{I}\right), E\left(\boldsymbol{\varepsilon} \boldsymbol{\varepsilon}_{\star}^{\boldsymbol{T}}\right)=\mathbf{0}$ and $\sigma^{2}=1$. The predicted results evaluated at $5 \%$ significance level are displayed in Figure 5. This is a well behaving model as evidenced by slowly diverging prediction and confidence intervals as compared to the trend only model solution.

Although the yearly predictions are useful for sea level studies as consilience values for satellite altimetry findings, their resolutions are not enough for addressing more practical problems such as coastal risk assessment, mitigation, and adaptation responses. The next section discusses another solution using high resolution monthly tide gauge data with markedly increased degree of freedom compared to the solution using yearly averages.

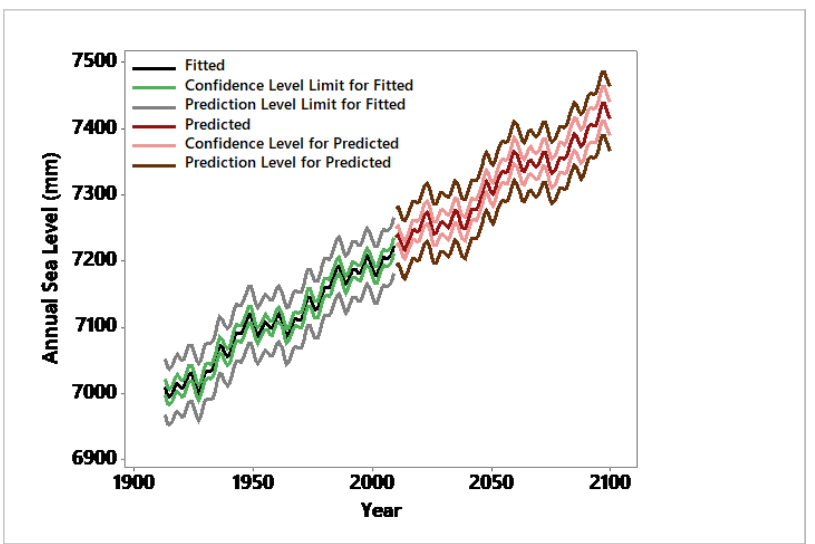

Figure 5: Adjusted (fitted) and predicted yearly sea levels with $95 \%$ confidence and prediction intervals. Reference epoch for the solution is 1961.

\section{Adjusted and predicted sea level rise using a harmonic model and monthly tide gauge records}

Following the same steps as before, another model was entertained this time using monthly Key West tide gauge records (Figure 1). This is a high-resolution predictive model inclusive of annual and semi-annual periodicities in sea level variations in addition to statistically significant interannual periodicities at $\alpha=0.05$ significance level, which were undetectable in the previous model because of the smoothing effect and smaller degrees of freedom of the yearly averaged tide gauge records (Table 3 ).

Annual and seasonal changes in tide gauge data are predominant in monthly data and induced mainly by the
Table 3: Solution statistics: the standar error of the disturbances, $\hat{\sigma}=44.45 \mathrm{~mm}$ and Adj $R^{2}=80.77$. Trend and estimated coefficients listed below are in $\mathrm{mm} / \mathrm{yr}$ and $\mathrm{mm}$ respectively. Only the model parameteres that are statistically significant $(\alpha=0.05)$, are used and listed in the final solution. Shaded parameters are rejected using first order autocorrelated disturbances instead of uncorrolated homogeneous disturbances.

\begin{tabular}{lll}
\hline Parameter & Estimate & St Error \\
\hline Constant & 7112.73 & 1.39 \\
Trend & 2.19 & 0.05 \\
C75 & -13.04 & 3.15 \\
S56 & -7.92 & 1.9 \\
C56 & 19.17 & 3.57 \\
S37 & -9.55 & 1.84 \\
C37 & -17.98 & 2.48 \\
C22 & -5.27 & 2.02 \\
S12.4 & -5.84 & 1.84 \\
C12.4 & 8.17 & 1.89 \\
S9 & 5.4 & 1.86 \\
C9 & -5.07 & 1.88 \\
S6 & -5.24 & 1.84 \\
C6 & 10.17 & 1.86 \\
S4 & 4.1 & 1.85 \\
Sann & 79.8 & 1.84 \\
Cann & 10.22 & 1.84 \\
Ssemi & -25.1 & 1.84 \\
Csemi & -30.21 & 1.84 \\
\hline
\end{tabular}

atmospheric pressure (the inverted barometer effect) not only in Key West station but also at other globally distributed tide gauge stations (Iz, 2017, 2018). Prediction intervals are accordingly large as shown in Figure 6 (confidence intervals were not included in the figure for clarity). In this solution, the trend estimate remained invariant, but its standard error improved markedly (from 0.81 down to 0.05) despite the larger $\hat{\sigma}^{2}(44.45$ vs. 20.53) and smaller Adj $\mathrm{R}^{2}$ values (80.77\% vs. $\left.90.82 \%\right)$. Improvements in the standard errors of trends are central in detecting the impact of climate change on sea level with statistical significance and must always be kept in mind using shorter time series such as those from satellite altimetry.

The solution and the predictions using monthly data are informative for a comparison with the yearly predictions in this study, yet the earlier assumptions regarding the disturbances are no longer valid for monthly tide gauge data. The disturbances of the Key West station's tide gauge exhibit a first order autoregressive process AR(1) because of the higher monthly resolution of the records, (Iz, 2014). Therefore, the disturbances stated in eq (18) are to be replaced by,

$$
\varepsilon_{t}=\rho \varepsilon_{t-1}+u_{t} \quad 0 \leq \rho<1
$$




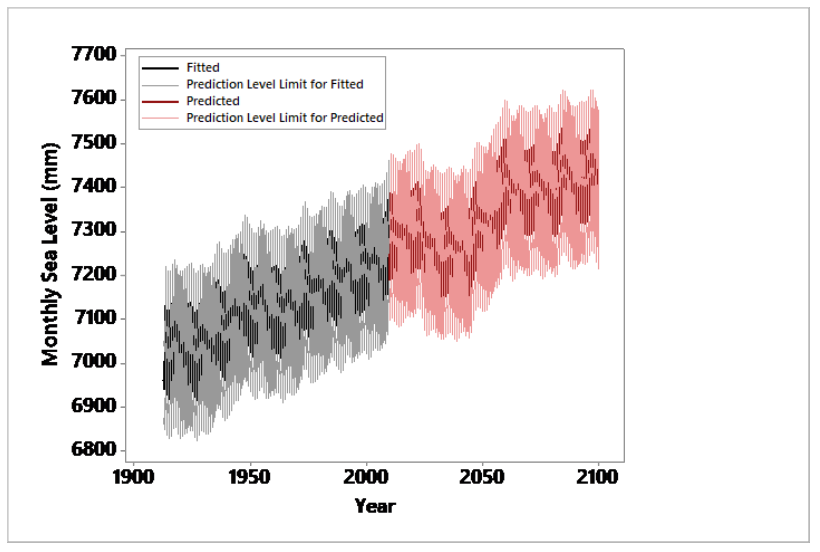

Figure 6: Adjusted (fitted) and predicted monthly sea levels. Confidence intervals were not included for clarity.

where $u_{t}$ is the white noise, $u_{t} \sim\left(0, \sigma_{u}^{2}\right)$. Although the first order autocorrelation, $\rho$, is relatively small for Key West tide gauge station disturbances $(\rho=0.4)$, it is still important in assessing the statistical significance of the model parameters for other tide gauge stations. For evidence, the parameters highlighted in bold in Table 3 were obtained from another solution, which accounted for the first order autocorrelation using Hildreth-Lu procedure (Iz, 2014). The effect of the first order autoregressive disturbances is to overestimate some of the model parameters which is evident by the larger number of parameters obtained using a statistical model with uncorrelated homogeneous disturbances. The results indicate that some of the borderline periodicities were rejected when autocorrelations are accounted for.

Despite this deficiency, the results are still informative revealing complexities ignored in projections and predictions using yearly tide gauge data. The formularies discussed in this study can also accommodate first order autocorrelations among monthly tide gauge records by modeling the variance/covariance matrix as demonstrated in Iz and Chen, (1999).

The formularies for the calculation of the prediction intervals also assume that , i.e. future disturbances are not correlated with the disturbances of the observed quantities, which is not the case here because of the AR(1) process. The formularies stated in Section (3) can be modified to accommodate for the correlations between the observed and predicted disturbances.

\section{Conclusion}

Empirical models, such as eq. (18) used in this study, to analyze sea level variations observed at globally distributed tide gauge stations during the last decade are now capable of generating precise and accurate sea level rise predictions. The utility of the explanatory climate models to project sea-level rise over the course of the 21st century must rely heavily on such predictive models not only for evaluating their performance in explaining the source of climate change but also for checking their resolutions. As discussed before, quantification and extension of sea level trends into the future enable assessing the distance between prediction and projection and thereby serve as a reality check for the relevance of the underlying theories. A very low predictability level for predictive models of sea level variations would lead to the development of new measures, alternative theories, new collected data, and new empirical approaches. An explanatory model that is close to a benchmark predictive model may suggest that our understanding of that phenomenon can only be increased marginally. If such a model is very far from the predictive benchmark, this outcome would imply that there are substantial practical and theoretical gains to achieve from further scientific development.

Moreover, empirical harmonic models accompanied by their prediction intervals can predict and assess short and long-term sea level changes in fine details. Prediction intervals, as demonstrated in this study, quantify the uncertainty of a single future observation from a population instead of the uncertainty of their expected values (confidence intervals), and thereby better serve the needs of coastal risk assessment, policy development, mitigation and adaptation responses.

Acknowledgement: I would like to gratefully acknowledge the two anonymous reviewers for their time and meticulous cross-checking of the formulas and the narrative in the manuscript.

\section{References}

Bibby, J. and H. Toutenburg, 1977, Prediction and improved estimation in linear models, John Wiley and Sons, New York.

Bindoff N.L., J. Willebrand, V. Artale, A, Cazenave, J. Gregory, S. Gulev, K. Hanawa, C. Le Quéré, S. Levitus, Y. Nojiri, C.K. Shum, L.D. Talley and A. Unnikrishnan (2007) Observations: Oceanic Climate Change and Sea Level. In: Climate Change 2007: The physical science basis. Contribution of working group i to the fourth assessment report of the intergovernmental panel on climate change [Solomon, S., D. Qin, M. Manning, Z. Chen, M. Marquis, K.B. Av- 
eryt, M. Tignor and H.L. Miller (eds.)]. Cambridge University Press, Cambridge, United Kingdom and New York, NY, USA.

Bray, D., H. Vomn Storch, 2009, Prediction or projection? The nomenclature of Climate Science, Science Communication Vol. 30 pp: 534-543.

Gabriele G., J. Feichter, Eds., 2011, Climate change and policy: The calculability of climate change and the challenge of uncertainty, Springer, 241 pgs.

Goldberger A. S., 1962, Best linear unbiased prediction in the generalized linear regression model, Journal of the American Statistical Association, Vol. 57, No. 298, pp.369-375.

IPCC, 2013: Climate Change 2013: The physical science basis. Contribution of working group I to the fifth assessment report of the Intergovernmental Panel on Climate Change [Stocker, T.F., D. Qin, G.-K. Plattner, M. Tignor, S.K. Allen, J. Boschung, A. Nauels, Y. Xia, V. Bex and P.M. Midgley (eds.)]. Cambridge University Press, Cambridge, United Kingdom and New York, NY, USA.

İz, H.B., 1992, Conditions for improved prediction when erroneous prior information is used about the expected values of stochastic parameters, Manuscripta Geodaetica, Vol.17, pp. 124-134.

İz H.B. and Y. Chen, 1999, VLBI Rates with First Order Autoregressive Disturbances, J. of Geodynamics, Vol. 28, No. 2-3, pp. 131-145.

İz H.B., 2014, Sub and super harmonics of the lunar nodal tides and the solar radiative forcing in global sea level changes, J. of Geod. Sci., Vol. 4, pp. 150-165.

İz H.B., 2015, More confounders at global and decadal scales in detecting recent sea level accelerations, J. of Geod. Sci., Vol., pp. 192-198.
Iz H.B., 2016, The effect of warming oceans at a tide gauge station, J. of Geod. Sci., Vol. 6, pp. 69-79.

İz H.B., 2017, The effect of regional sea level atmospheric pressure to sea level variations at globally distributed tide gauge stations with long records, J. Geod. Sci. Vol. 8, pp. 55-71.

İz H.B., 2018: Is the global sea surface temperature rise accelerating? Geodesy and Geodynamics, 1-7. https://doi.org/10.1016/j.geog. 2018.04.002.

Keeling, C. D., and Whorf T. P., 1997, Possible forcing of global temperature by oceanic tides. Proc. Natl. Acad. Sci., 94, 8321-8328.

Mastrandrea, M.D., C.B. Field, T.F. Stocker, O. Edenhofer, K.L. Ebi, D.J. Frame, H. Held, E. Kriegler, K.J. Mach, P.R. Matschoss, G.K. Plattner, G.W. Yohe, and F.W. Zwiers, 2010: Guidance Note for Lead Authors of the IPCC Fifth Assessment Report on Consistent Treatment of Uncertainties. Intergovernmental Panel on Climate Change (IPCC). Available at http://www.ipcc.ch.

Munk, W., Dzieciuch M., Jayne S., 2002, Millennial climate variability: Is there a tidal connection? J. Climate, 15, 370-385.

Nerem R. S., B. D. Beckley, J. T. Fasullo, B. D. Hamlington, D. Masters and G. T. Mitchum, 2018, Climate-change-driven accelerated sealevel rise detected in the altimeter era. PNAS, 1-4.

Permanent Service for Mean Sea Level, 2016, Tide Gauge Data, Retrieved February 2016 from http://www.psmsl.org/data/ obtaining/.

Toutenburg, H., 1982, Prior information in linear models, John Wile and Sons, New York. 